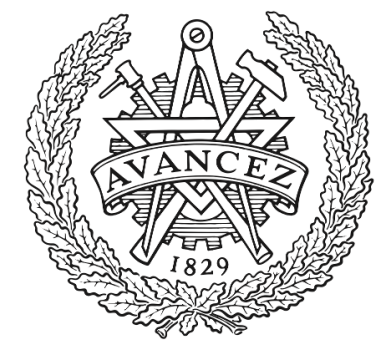

CHALMERS

UNIVERSITY OF TECHNOLOGY

\title{
Different gap waveguide slot array configurations for mmwave fixed beam antenna application
}

Downloaded from: https://research.chalmers.se, 2023-04-26 14:47 UTC

Citation for the original published paper (version of record):

Uz Zaman, A., Kildal, P. (2016). Different gap waveguide slot array configurations for mmwave fixed beam antenna application. 10th European Conference on Antennas and Propagation, EuCAP 2016; Davos; Switzerland; 10 April 2016 through 15 April 2016: Art. no. 7481541-. http://dx.doi.org/10.1109/EuCAP.2016.7481541

N.B. When citing this work, cite the original published paper. 


\title{
Different Gap Waveguide Slot Array Configurations for mmWave Fixed Beam Antenna Application
}

\author{
Ashraf Uz Zaman ${ }^{1}$, Per-Simon Kildal ${ }^{1}$ \\ ${ }^{1}$ Signals and Systems Department, Chalmers University of Technology, Göteborg, Sweden, \\ E-mail: zaman@chalmers.se ; per-simon.kildal@chalmers.se
}

\begin{abstract}
In this work, we present several gap waveguide planar array antennas for millimeter wave point to point communication systems. The antennas are designed using groove gap waveguide, ridge gap waveguide and inverted microstrip gap waveguide technology. All these three antenna designs are based on the cavity-backed slots as the core radiating elements. There exists a coupling slot in the cavity layer which allows the excitation of the radiating slot elements using three different types of feeding waveguide section. The designed three antennas have $16 \times 16$ radiating slot elements and these antennas operate over $15 \%$ relative bandwidth from $57-66 \mathrm{GHz}$ frequency range with $-13 \mathrm{~dB}$ reflection coefficient. The simulated directivity for these slot arrays are about $33.3 \mathrm{dBi}$ at the center of the band.
\end{abstract}

Index Terms-Cavity backed slot-array, waveguide slot-array, corporate feed network.

\section{INTRODUCTION}

Fixed wireless systems operating in the millimeter wave frequency range are extremely attractive for high data rate wireless broadband. Such systems require novel antenna architectures both for the base station and end user terminals. In particular, the outdoor base stations need compact high gain and high efficiency antennas to compensate for the free space path loss, especially in the $60-\mathrm{GHz}$ band where a strong absorption peak occurs due to oxygen molecules. Therefore, for such applications, low-loss planar antenna solutions are very attractive. Waveguide slot array antennas are expected to provide high efficiency and high gain even at mm-Wave frequency range due to lower losses in antenna feed networks [1-2]. Waveguide slot array antennas can be series fed type or parallel fed type. Series-fed slot array antennas have simple geometry but suffer from narrow operational bandwidth due to long-line effect [3-4]. On the other hand, multiple layer cavitybacked slot array antennas can have higher efficiency as well as wider bandwidth. Antennas around $75-80 \%$ efficiency and $10 \%$ relative bandwidth have been described in [5-6]. But the key challenges with multi-layer antenna structure are high fabrication cost and manufacturing complexity to achieve good electrical contacts among the feed layer, cavity layer and radiating slot layer.

To overcome this problem of good electrical contact and problems associated with mechanical assembly, the gap waveguide technology can be employed successfully. The gap waveguide technology presented in [7-8] uses the cut-off of a PEC-PMC parallel-plate waveguide configuration to control desired electromagnetic propagation between the two parallel plates without the requirement of the electrical contact. This is quite advantageous for the mechanical assembling of mmWave antennas when tolerance in fabrication process becomes a key factor at such high frequencies. Also, the Q-factor analysis confirms that the losses in ridge gap waveguide and groove gap waveguide structures are comparable to that of standard rectangular waveguide [9]. Therefore, the feed network losses will be quite low for gap waveguide antennas and the total efficiency of gap waveguide array antennas will be quite high. To date, several gap waveguide antennas have been manufactured at different frequency bands [10-14]. Also the gap waveguide technology is very suitable for RF packaging [15-16], which plays an important role in integrating $\mathrm{RF}$ electronics with the antenna.

In this work, we present different high gain gap waveguide antenna configurations realized by groove gap, ridge gap and inverted microstrip gap waveguide feeding network. All these antennas have been designed at mmWave frequency range.

\section{ANTENNA CONFIGURATIONS}

In real gap waveguide structure, the bottom periodic textured has high enough surface impedance to create a stopband over which no parallel-plate modes can propagate. However, the textured AMC surface must also incorporate guiding structures in the form of ridges, grooves or strips to form the complete waveguide. As a result of the stopband, the electromagnetic waves can propagate along these guiding stuctures without leaking away in other directions. Different configurations of gap waveguide structures are shown in fig.1.
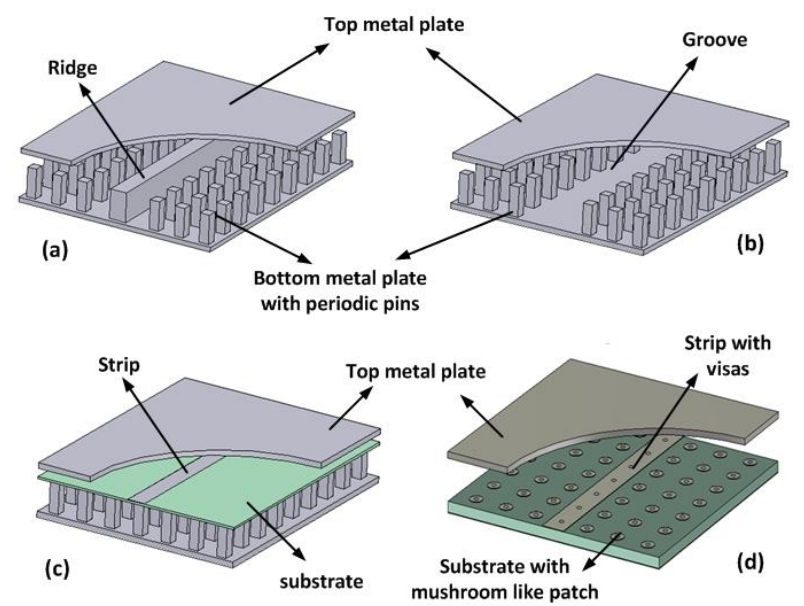

Fig. 1 Different Gap waveguide geometries: a) Ridge gap waveguide, b) Groove gap waveguide, c) Inverted- microstrip gap waveguide, d) Microstripridge gap waveguide. 

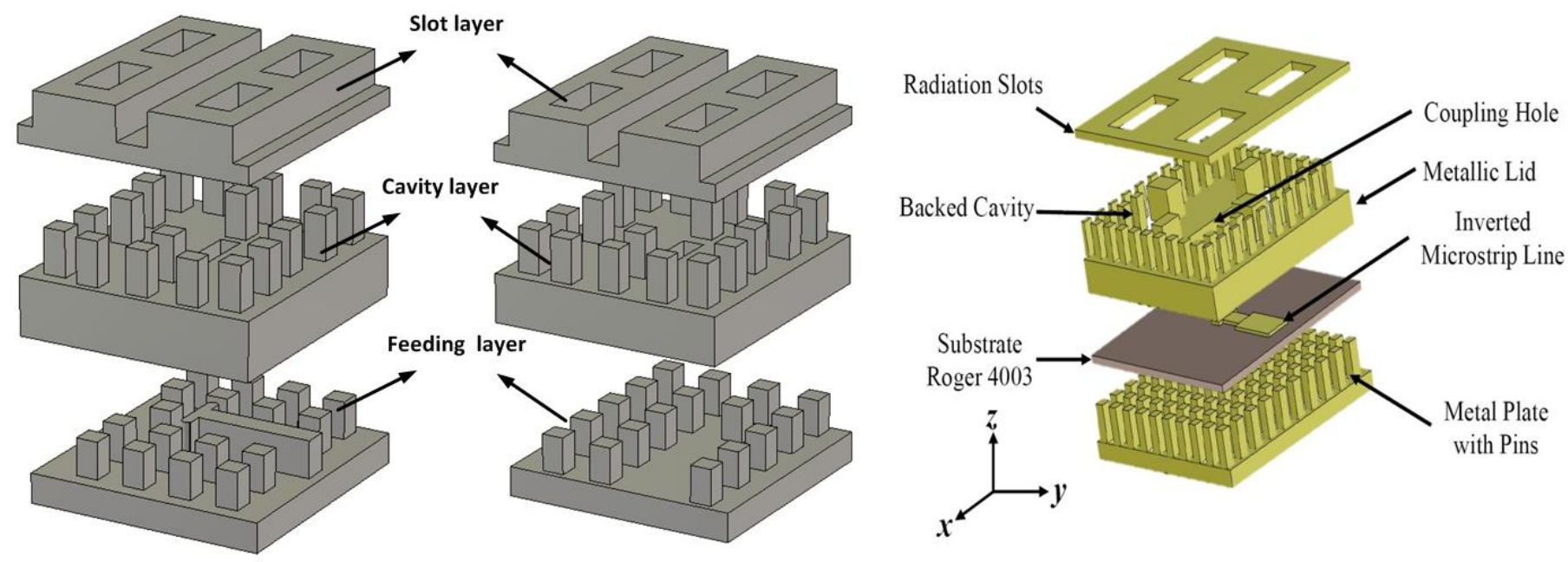

Fig.2 The schematics of the subarray used in the antenna designs.

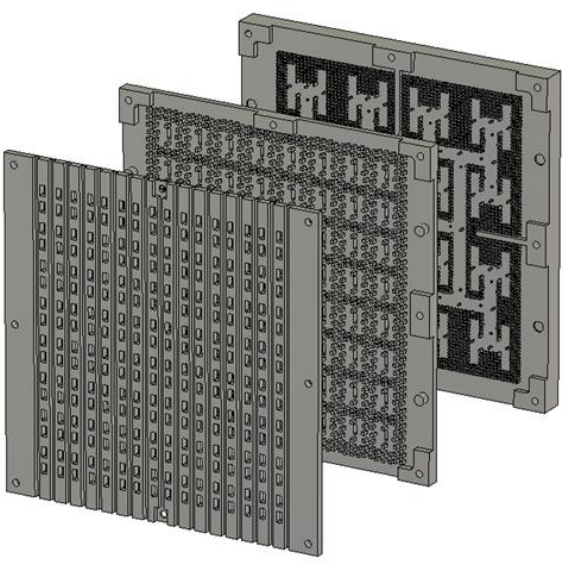

Groove gap waveguide slot array

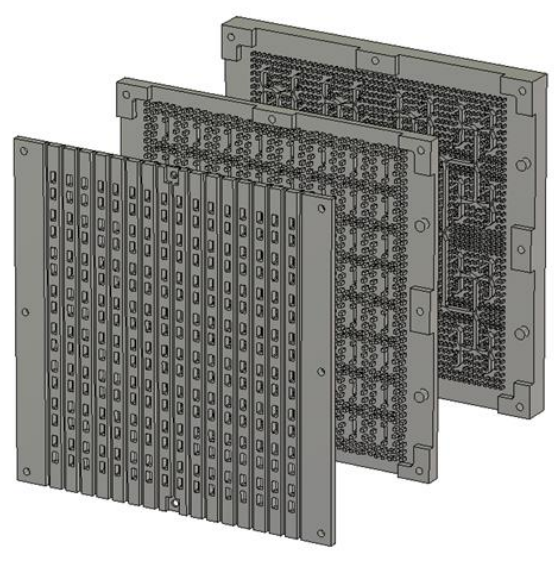

Ridge gap waveguide slot array

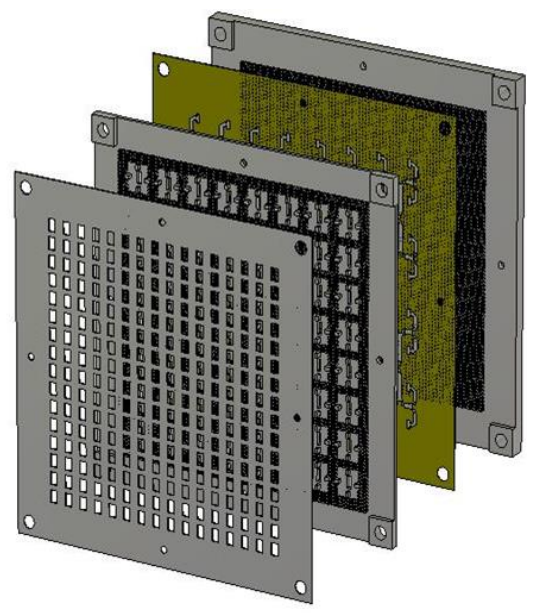

Inverted microstrip gap waveguide slot array

Fig. 3 The schematics of the three $16 \times 16$ slot array designed in gap waveguide technology.

The intention in this work is to present several high gain antenna designs based on different gap waveguide configuration. The subarray unit used in all these designs is quite similar. The subarrays are shown in fig. 2. As shown in fig.2, the antenna subarray consists of cavity backed slot layer in which the cavity is excited by feed waveguide by means of a coupling slot. The size of the subarray is about $8 \times 8 \mathrm{~mm}^{2}$.

Based on these subarrays, three big antenna arrays have been designed. The $16 \times 16$ element array antennas are shown in fig.3. The feed network for these three antennas have been designed based on three gap waveguide topology such as groove gap waveguide, ridge gap waveguide and inverted microstrip. The corporate feeding networks for all three designs are based on 3-dB T-junctions cascading after one another. In this way, all the subarrays in the big array can be excited in parallel with same phase and same amplitude which is needed for a lower sidelobe level. All these antennas are excited with a WR-15 rectangular waveguide and transitions from groove gap waveguide, ridge gap waveguide and inverted microstrip waveguide to rectangular waveguide have been designed. The conventional methods for manufacturing such antenna are metal milling and metal molding. But there are many modern low cost manufacturing technologies that will suit such planar surfaces with texture, such as die sink Electrical Discharge Manufacturing (EDM), Electron Beam Melting (EBM), multilayer die pressing, and 3D screen printing. Therefore, such antennas designed in gap waveguide technology have a large potential for millimeter wave applications.

\section{MATCHING BANDWIDTH AND RADIATION PATTERNS}

All these three antennas are designed to have good $\mathrm{S}_{11}$ over the band of interest from $57-66 \mathrm{GHz}$. The simulated $S_{11}$ for the ridge gap waveguide slot array, groove gap slot array and inverted microstrip ridge gap waveguide array are shown in fig. 4. 


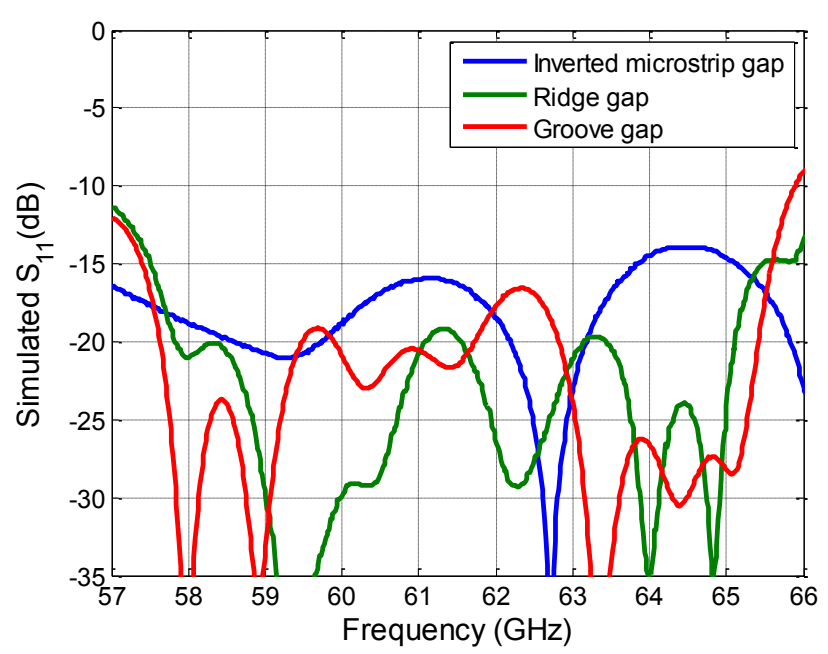

Fig.4 Simulated $\mathrm{S}_{11}$ for three gap waveguide array antenna.

The simulated far-field patterns in E-plane, H-plane and $45^{\circ}$ plane for the ridge gap waveguide array antenna at 58, 62, and $66 \mathrm{GHz}$ are shown in fig.5(a) and fig.5(b) and fig.5(c) respectively. The patterns for the groove gap waveguide array are very similar to the patterns shown in fig. 5 as the radiating layers for both these antennas are very identical. As expected, we find that the E-plane patterns have some grating lobes. But the level still remains below the acceptable limit. The reason of the grating lobe is attributed to the subarray dimensions, which are more than one wavelength in both $\mathrm{E}$ and H-plane of the antennas.

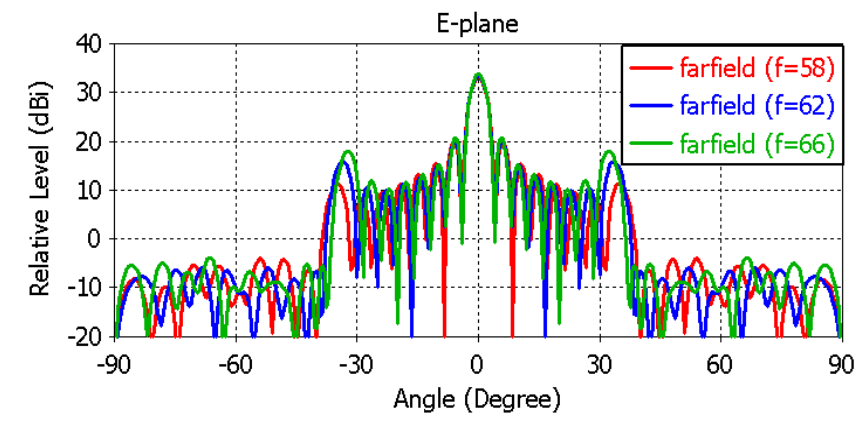

Fig.5 (a) Simulated E-plane patterns for the $16 \times 16$ element ridge gap waveguide array antenna.

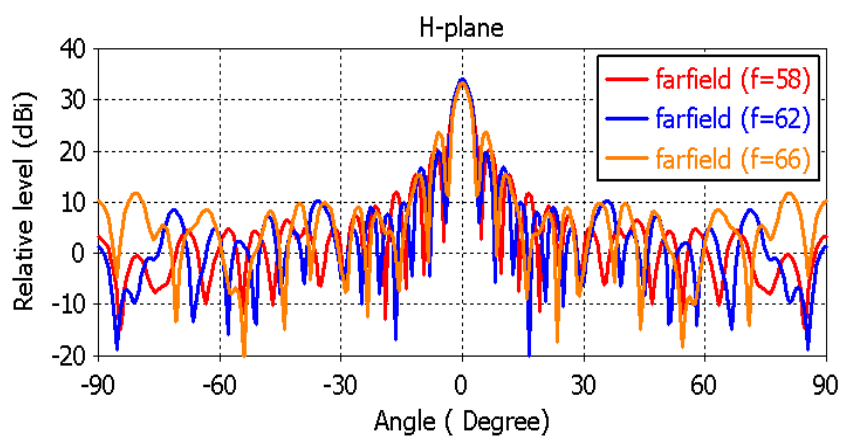

Fig.5 (b) Simulated H-plane patterns for the $16 \times 16$ element ridge gap waveguide array antenna.

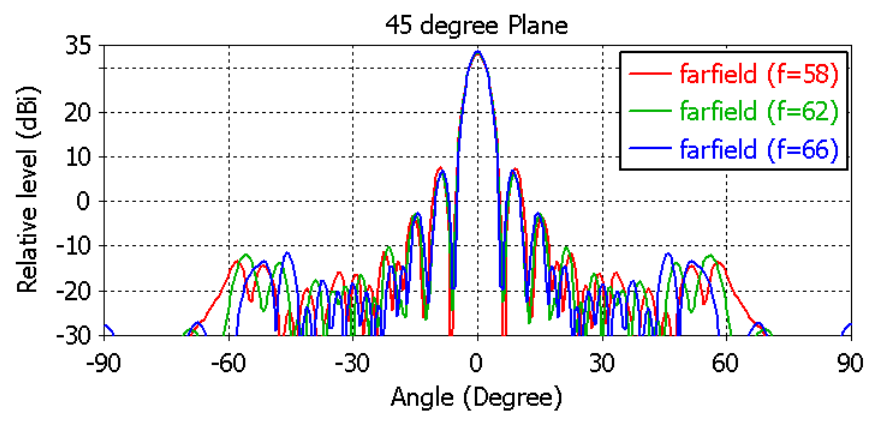

Fig.5 (c) Simulated $45^{\circ}$ plane patterns for the $16 \times 16$ element ridge gap waveguide array antenna.

The radiation patterns for the inverted microstrip gap waveguide is presented in fig.6(a) and fig.6(b). The sidelobe levels in this antenna are even lower than the other two antennas. This is because; the subarray size is little bit smaller for this antenna.

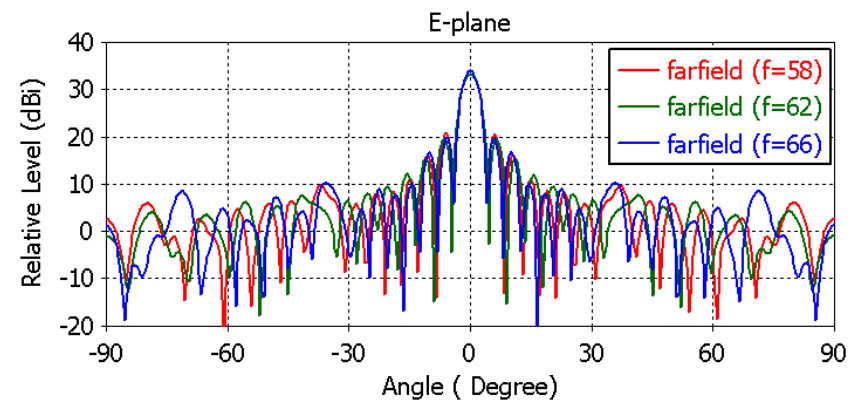

Fig.6 (a) Simulated E-plane patterns for the $16 \times 16$ element inverted microstrip gap waveguide array antenna.

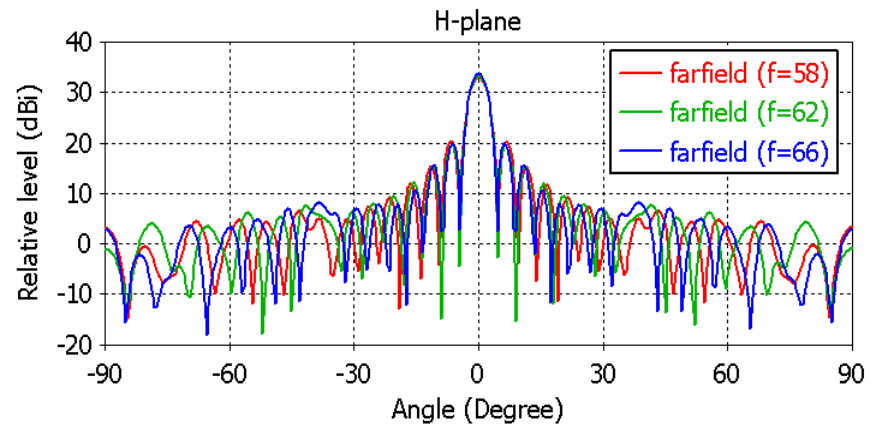

Fig.6 (b) Simulated H-plane patterns for the $16 \times 16$ element inverted microstrip gap waveguide array antenna.

The simulated cross-polar levels for all these three antennas are quite low, in the order of $-35 \mathrm{~dB}$. This is because, the slot dimensions are chosen in such a way that the higher order modes are not excited in the radiating slots. The simulated directivity of these $16 \times 16$ slot-array antennas are found to be around $33.3 \mathrm{dBi}$ at the center of the band. The aperture efficiency is about $90 \%$ for all these three antennas. Here in this work, only the simulated directivity for the ridge gap waveguide antenna array is shown in figure 7 . The directivity of the groove gap waveguide and inverted microstrip ridge gap waveguide looks very similar also. 


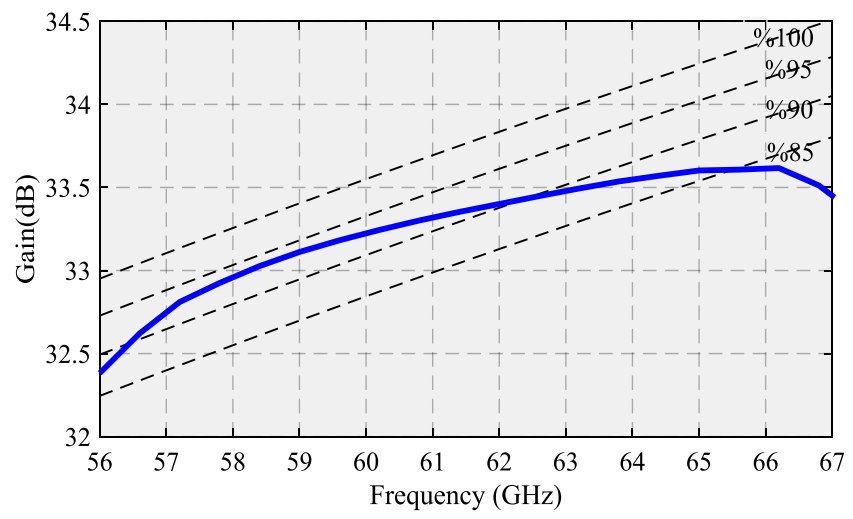

Fig.7 Simulated directivity for the $16 \times 16$ element ridge gap waveguide array antenna.

\section{CONCLUSION}

We present three multilayer fixed beam slot array antenna design based on gap waveguide technology in this work. The designed antennas have good impedance bandwidth and also good radiation patterns over the band of interest at $60 \mathrm{GHz}$. The main feature of these gap waveguide antennas is the flexibility in mechanical assembly which will allow low cost manufacturing techniques to be used in slot array antenna manufacturing and will lower the overall cost of the mmWave modules.

\section{Acknowledgment}

This work has been supported by the European Research Council (ERC) via an advanced investigator grant ERC-2012ADG_20120216 and by the Swedish Governmental Agency for Innovation Systems (VINNOVA) within the VINN Excellence Center Chase.

\section{REFERENCES}

[1] J. Hirokawa and M. Ando, "Efficiency of 76-GHz post-wall waveguidefed parallel-plate slot arrays," IEEE Trans. Antenna Propag., vol.48, no. 11, pp. 1742-1745, Nov. 2000.

[2] J. Hirokawa and M. Ando, "Single-layer feed waveguide consisting of posts for plane TEM wave excitation in parallel plates", IEEE Trans. Antennas Propag., vol. 46, no. 5, pp. 625-630, May 1998.

[3] YKimura, T.Hirano, J.Hirokawa and M.Ando, "Alternating-phase fed single-layer slotted waveguide arrays with chokes dispensing with narrow wall contacts," IEE Proc.-Microw. Antennas Propag, Vol. 148, No 5, October 2001.

[4] N.Goto, "A Planar Waveguide Slot Antenna of Single Layer Structure ," IEICE Tech. Report, AP 88-39,1988.

[5] Y. Miura, J. Hirokawa, M. Ando, Y. Shibuya, and G. Yoshida, "Doublelayer full-corporate-feed hollow-waveguide slot array antenna in the 60 GHz-band," IEEE Trans. Antennas and Propag., vol. 59, no. 8, pp. 2844-2851, Aug. 2011.

[6] B. Lee, K. Jung and S.Yang, "High Efficiency Planar Slot Array Antenna with a Single Waveguide-fed Cavity-backed Subarray," Microw. Opt.Tech. Letter, vol. 43, no. 3, pp. 228-231, Nov.2004.

[7] P.-S. Kildal, A. Uz Zaman, E. Rajo-Iglesias, E. Alfonso and A. ValeroNogueira, "Design and experimental verification of ridge gap waveguides in bed of nails for parallel plate mode suppression," IET Microwaves, Antennas \& Propagation, vol.5, iss.3, pp. 262-270, March 2011.
[8] A. Uz Zaman, P.-S. Kildal, M. Ferndahl and A. kishk, "Validation of Ridge Gapwaveguide Performance by Using In-house TRL Calibration Kit,"4th European Conference on Antennas and Propagation, Barcelona, April, 2010.

[9] Elena Pucci, Ashraf Uz Zaman, Eva Rajo-Iglesias, Per-Simon Kildal, and Ahmed Kishk, "Study of Q-factors of ridge and groove gap waveguide resonators," IET Microwaves, Antennas \& Propagation, vol.7, iss.11, pp. 900-908, August 2013.

[10] E. Pucci, E. Rajo-Iglesias, J. L. Vazquez-Roy, and P. S. Kildal, "Planar Dual-Mode Horn Array With Corporate-Feed Network in Inverted Microstrip Gap Waveguide," IEEE Trans. Antennas Propag. , vol. 62, no. 6, pp. 3534-3542, 2014.

[11] A. Razavi, P.-S. Kildal, X. Liangliang, E. Alfonso, and H. Chen, "2x2slot Element for $60 \mathrm{GHz}$ Planar Array Antenna Realized on Two Doubled-sided PCBs Using SIW Cavity and EBG-type Soft Surface fed by Microstrip-Ridge Gap Waveguide," IEEE Trans. Antennas Propag. , vol. 62, no. 9, pp. 4564-4573, Sep. 2014.

[12] A. U. Zaman and P. S. Kildal, "Slot antenna in ridge gap waveguide technology," in Antennas and Propagation (EUCAP), 2012 6th European Conference on, 2012, pp. 3243-3244.

[13] B. Cao, H. Wang, and Y. Huang, "W-band High Gain TE220-Mode Slot Antenna Array with Gap Waveguide Feeding Network," Accepted for publication in IEEE Antennas and Wireless Propagation Letters.

[14] A. Vosoogh and P.-S. Kildal, "Corporate-Fed Planar 60 GHz Slot Array Made of Three Unconnected Metal Layers Using AMC pin surface for the Gap Waveguide", Accepted for publication in IEEE Antennas and Wireless Propagation Letters.

[15] A. Kishk, A. Uz Zaman, and P.-S. Kildal, "Numerical Prepackaging with PMC lid - Efficient and Simple Design Procedure for Microstrip Circuits including the Packaging," ACES Applied Computational Society journal, vol. 27, no.5, pp. 389-398, May 2012.

[16] E. Rajo-Iglesias, P. S. Kildal, A. U. Zaman, and A. Kishk, "Bed of Springs for Packaging of Microstrip Circuits in the Microwave Frequency Range," Components, Packaging and Manufacturing Technology, IEEE Transactions on, vol. 2, pp. 1623-1628, 2012. 